\title{
Ferrous Xylenol Gel Measurements for 6 and 10 MV Photons in Small Field Sizes
}

\author{
Lucas N. de Oliveira ${ }^{a}$, Carmen S. Guzmán Calcina ${ }^{a, b}$, Marco A. Parada ${ }^{a}$, Carlos E. de Almeida ${ }^{b}$, and Adelaide de Almeida ${ }^{a}$ \\ ${ }^{a}$ Departamento de Física e Matemática, Faculdade de Filosofia, \\ Ciências e Letras de Ribeirão Preto, Universidade de São Paulo, \\ Av. Bandeirantes 3900, Monte Alegre, 14015-400, Ribeirão Preto, SP, Brazil \\ ${ }^{b}$ Laboratório de Ciências Radiológicas, Universidade do Estado de Rio de Janeiro, \\ Rua São Francisco Xavier, 524, Rio de Janeiro, RJ, Brazil
}

Received on 13 June, 2007

\begin{abstract}
The Fricke dosimeter is a ferrous sulfate aqueous solution that, when irradiated, oxidizes the $\mathrm{Fe}^{2+}$ ions to $\mathrm{Fe}^{3+}$. This new concentration, generally determined through spectrophotometry, is directly proportional to the ionizing radiation absorbed energy. The Fricke Xylenol Gel dosimeter (FXG) was developed through the incorporation of swine skin gelatin and xylenol orange. These modifications provided better signal stability and sensitivity for lower absorbed dose measurements, such as those used in radiotherapy. In this work FXG samples were irradiated with absorbed doses of $2 \mathrm{~Gy}$, from $6 \mathrm{MV}$ and $10 \mathrm{MV}$ photons, using small field sizes geometry for dosimetric parameters determination. All the FXG dosimeter readings were accomplished with our specially developed spectrophotometer, using a narrow light beam at the wavelength of $585 \mathrm{~nm}$, where the highest absorbance sensitivity occurs. From our results, we can confirm not only that the FXG dosimetric system (FXG plus a high lateral spatial resolution spectrophotometer) can be used for general dosimetry, but as well for small field size dosimetry of interest in radiosurgery.
\end{abstract}

Keywords: Fricke Xylenol Gel dosimeter; Physical parameters; Linear accelerator

\section{INTRODUCTION}

Radiotherapy uses ionizing radiation to eliminate or neutralize cancer cells. It is important to previously determine the absorbed dose distribution in the target volume and in adjacent tissues to guarantee the prescribed treatment. For the treatment planning, the dosimetric parameters dependent on the beam energy, target composition and irradiation geometry are necessary to calculate how the absorbed dose will be distributed in the patient target volume (tumor). The parameters percentage depth dose, beam profile and output factor, once obtained, are used in the service routine for patient absorbed dose calculations. In this way, they have to be confirmed routinely by medical physicists for treatment Quality Assurance. The selected dosimetric system must be accurate, reproducible and linear with the absorbed dose. In the Fricke Xylenol Gel (FXG) chemical system the $\mathrm{Fe}^{3+}-$ Xylenol complex concentration, measured spectrophotometrically, is directly proportional to the absorbed dose [1-4]. The FXG dosimeter characteristics make it suitable for radiotherapy as: broad linear dependence on the absorbed dose up to $30 \mathrm{~Gy}$ for ${ }^{60} \mathrm{Co}$ radiation $[5,6]$, effective atomic number of 7.75 and density of $1.05 \mathrm{~g} / \mathrm{cm}^{3}$ near to 7.64 and $1.04 \mathrm{~g} / \mathrm{cm}^{3}$, respectively, for soft tissue. The first chemical dosimeter, prepared by Fricke in 1921 (ferrous sulfate, sulphuric acid and water), has been improved adding or substituting the originals components. The FXG is prepared by the addition of swine skin gelatin and xylenol orange to the original Fricke solution. These dosimeters provide spatial resolution comparable to those of the film dosimeters and natural oxidation lower than that, from the original Fricke solution [5]. In this work, the FXG dosimeter was used to determine output factors, percentage depth dose curves (PDD) and field profiles for $6 \mathrm{MV}$ and $10 \mathrm{MV}$ photons. The FXG results were compared with those determined with X-OMAT V film and $0.1 \mathrm{~cm}^{3}$ and 0.125 $\mathrm{cm}^{3}$ ionization chambers (IC). From the comparison of these data sets, obtained with our FXG dosimetric system with those from ion chambers and film, we show that our FXG system is an adequate, and in some important aspects, a superior dosimetric system for small field size measurements in radiotherapy.

\section{MATERIALS AND METHODS}

\section{General Description}

The small field size relative basic data sets were obtained with photons from a linear accelerator (Varian/ Clinac 2100) for photon energies of $6 \mathrm{MV}$ and $10 \mathrm{MV}$. The FXG was contained in $1.0 \times 1.0 \times 3.0 \mathrm{~cm}^{3}$ acrylic cuvettes (three for each measured value) inserted in the phantoms PI and PIII shown in Fig. 1 and the ionization chamber, was inserted in the phantom PII. Fig. 1 presents the acrylic phantoms in sandwich configurations of $15 \times 15 \mathrm{~cm}^{2}$ PMMA plates, with $2 \mathrm{~cm}$ below and $1.5 \mathrm{~cm}$ or $2.5 \mathrm{~cm}$ above, with a PMMA plate for dosimeter insertion. The dosimeters were irradiated at a source surface distance (SSD) of $100 \mathrm{~cm}$ for square field sizes, defined by the Linac collimator. Absorbed doses of $1 \mathrm{~Gy}$ were used for output factors and field profiles measurements and of $2 \mathrm{~Gy}$ for PDD measurements.

\section{FXG Dosimeter}

The FXG dosimeters were prepared from swine skin gelatin 300 Bloom (Aldrich), sulfuric acid, ferrous ammonium sulphate and xylenol orange $[5,6]$. The gelatin solution was inserted in $1 \times 1 \times 3 \mathrm{~cm}^{3}$ cuvettes and the FXG optical transmission measurements were done through a $2 \mathrm{~mm}$ diameter 
(A)

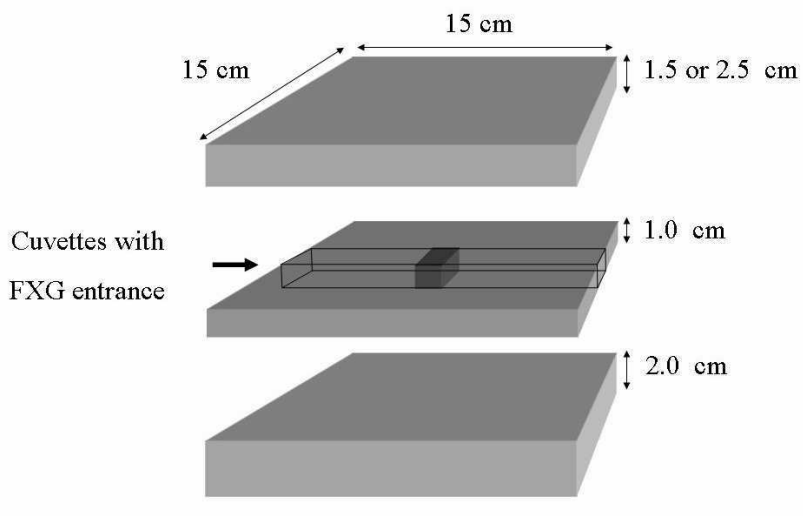

PI

(B)

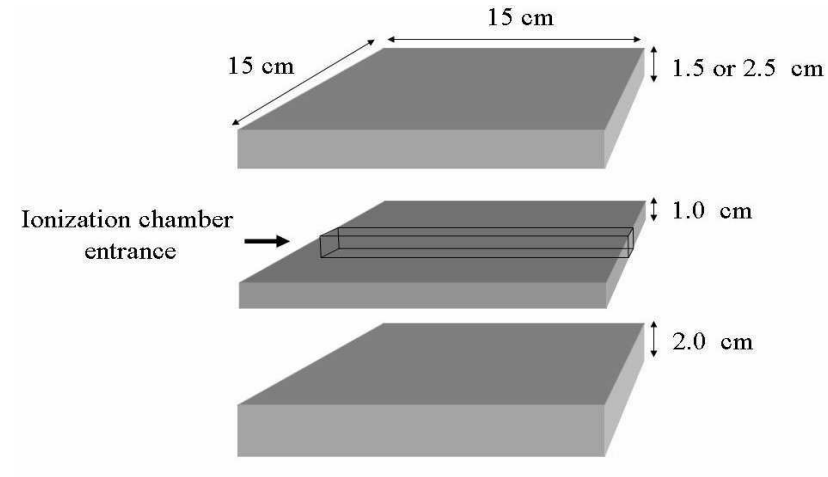

PII

(C)

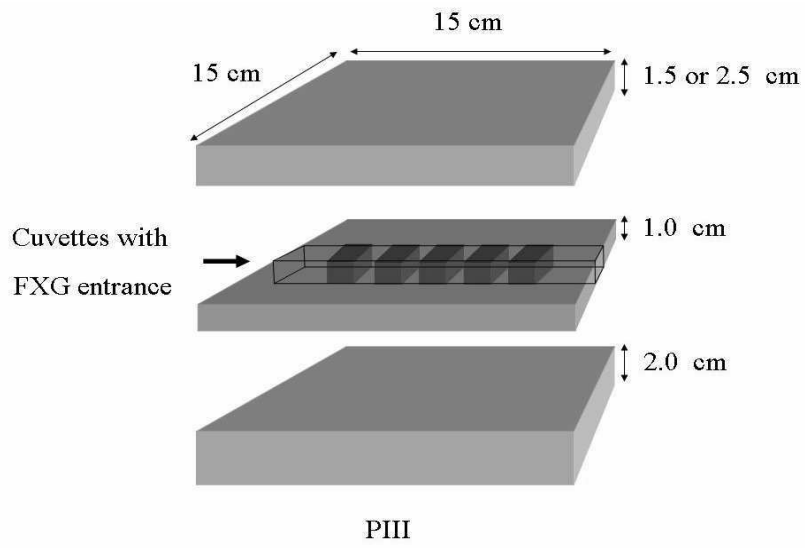

FIG. 1: Acrylic phantoms for the percentage depth dose, calibration curve, output factor and profile measurements. a) PI with plate for cuvette insertion, b) PII with plate for IC insertion and c) PIII for cuvettes insertion.

and $1 \mathrm{~cm}$ of length through the dosimeter, optical path, with a portable spectrometer (Vary-Varian/Ultropec 2100/79500) at $585 \mathrm{~nm}$.

\section{Calibration Curve}

The FXG dosimeter calibration curve was obtained, using a ${ }^{60} \mathrm{Co}$ source (Siemens/Theratron $780 \mathrm{C}$ ) and a $0.125 \mathrm{~cm}^{3}$ ionization chamber, connected to a (PTW-Freiburg/M31010) electrometer both from the secondary standards laboratory (Laboratório de Ciências Radiológicas-UERJ). The FXG dosimeters were placed in phantom PI shown in Fig. 1 at a SSD of $80 \mathrm{~cm}, 10 \times 10 \mathrm{~cm}^{2}$ field size with a build-up thickness of $0.5 \mathrm{~cm}$ PMMA. Five nominal absorbed dose values were selected in the interval from 0.5 Gy to 6.0 Gy. For each selected dose, three FXG dosimeters were irradiated and their optical transmission values averaged. The relative absorbance was calculated from the logarithm of the ratio of transmission values of each FXG dosimeter, before and after irradiation and the reproducibility was less than $2.5 \%$.

\section{Output Factor}

The output factor measurements [7] were obtained in the PMMA phantoms with FXG detectors and with the $0.125 \mathrm{~cm}^{3}$ IC for an absorbed dose of $1 \mathrm{~Gy}, \mathrm{SSD}$ of $100 \mathrm{~cm}$, a $1.5 \mathrm{~cm}$ build-up plate for $6 \mathrm{MV}$ photons and a $2.5 \mathrm{~cm}$ build-up plate for $10 \mathrm{MV}$ photons. Single FXG dosimeters were inserted in the phantom PI and centered in the square field sizes of $1 \times 1$ $\mathrm{cm}^{2} ; 2 \times 2 \mathrm{~cm}^{2} ; 2.5 \times 2.5 \mathrm{~cm}^{2} ; 4 \times 4 \mathrm{~cm}^{2} ; 5 \times 5 \mathrm{~cm}^{2} ; 7 \times 7 \mathrm{~cm}^{2}$; $8 \times 8 \mathrm{~cm}^{2}$ and $10 \times 10 \mathrm{~cm}^{2}$. Three of such FXG dosimeters were irradiated in the same conditions and their absorbed dose values averaged, for each field size and photon energy. The irradiation conditions with the ionization chamber were the same cited, but with the chamber positioned in the center of the phantom PII.

The relative output factor is expressed as the ratio of the absorbed dose at the center of a selected field size to that at the center of a larger reference field size $\left(10 \times 10 \mathrm{~cm}^{2}\right)$, both measured at the maximum dose depth in the same medium.

$$
R=\frac{D(r)}{D(10)}
$$

where: $D(r)$ and $D(10)$ are respectively the dosimeter response for the selected field size and the reference field size.

\section{Percentage Depth Dose}

An absorbed dose at a depth $d$ can be derived from the beam geometrical parameters, such as distance from the source and field size, as well the attenuation in the media. The depth dose can be represented through the following expression [8]:

$$
D_{d}=D_{m} \cdot\left(\frac{S S D+d_{m}}{S S D+d}\right)^{2} \cdot B \cdot e^{-\mu d}
$$

where: $D_{d}$ is the dosimeter response at a particular depth and $D_{m}$ is that at the maximum dose depth, $S S D$ is the source skin 
distance, $d_{m}$ is the build-up depth, $\mathrm{d}$ is a selected depth at the beam central axis $\left(d>d_{m}\right), B$ is the scatter factor and $e^{-\mu d}$ is the attenuation factor related to the material media and depth selected.

The PDD were obtained for three square field sizes $(1 \mathrm{x}$ $1 \mathrm{~cm}^{2} ; 3 \times 3 \mathrm{~cm}^{2}$ and $5 \times 5 \mathrm{~cm}^{2}$ ) using PI and PII phantoms, respectively for FXG and $0.125 \mathrm{~cm}^{3}$ IC dosimeters. The dosimeters when positioned in build-up depth, were exposed to an absorbed dose of $2 \mathrm{~Gy}$ from $6 \mathrm{MV}$ and $10 \mathrm{MV}$ photons at a SSD of $100 \mathrm{~cm}$. The dosimeters were irradiated at several depths by adding acrylic plates. For each depth, three FXG and IC exposures were performed. The PDD was obtained from the measurements, following the relation:

$$
P D D=\frac{D_{d}}{D_{m}} \cdot 100
$$

The PDD measurements in acrylic were converted [9] for those in water through the Eq.(4) and were compared with the measurements from literature $[10,11]$.

$$
d_{a}=d_{w}(\bar{\mu})_{a}^{w}
$$

where: $d_{w}$ and $d_{a}$ are the equivalent depths, respectively in water and acrylic and $(\bar{\mu})_{a}^{w}$ is the mean linear absorption coefficient ratio (water/acrylic).

\section{6. $\quad$ Field Profile}

The field profiles were determined at $1.5 \mathrm{~cm}$ and $2.5 \mathrm{~cm}$, the maximum dose depths, respectively for $6 \mathrm{MV}$ and $10 \mathrm{MV}$ photons, SSD of $100 \mathrm{~cm}$ for an absorbed dose of 2 Gy for $3 \times 3 \mathrm{~cm}^{2}$ and $5 \times 5 \mathrm{~cm}^{2}$ field sizes, using the FXG and film dosimeters. For the FXG profile irradiations, the phantom PIII was used and the FXG cuvettes were inserted in amounts of 9 and 11, to provide the expected field size lengths for the measurements. Three FXG dosimeters were used for each profile point, whose readings were averaged and normalized for the maximum absorbed dose value (in the center of the field). The same conditions were used for the film irradiations, but the films were inserted between the acrylic slices and irradiated with doses of $0.7 \mathrm{~Gy}$ for $6 \mathrm{MV}$ and $10 \mathrm{MV}$ photons. After development, they were read with a densitometer (PTW-Freiburg/DensinXauto/79115) and the FXG and film data were normalized to their central values, obtained for each field size and energy.

\section{RESULTS AND DISCUSSION}

\section{Calibration Curve}

In Fig. 2 the FXG relative optical absorbance versus absorbed dose is shown in the interval of $0.5 \mathrm{~Gy}-6.0 \mathrm{~Gy}$. This absorbed dose calibration curve is linear with a differential absorbance sensitivity of $0.037 \mathrm{~Gy}^{-1}$. The linear interval tested is consistent with the results cited in the literature $[5,6,12,13]$ and is adequate for radiotherapy purposes. The basic data set that follows is relative to each dosimeter response at a given position and depth, and did not use the calibration curves.

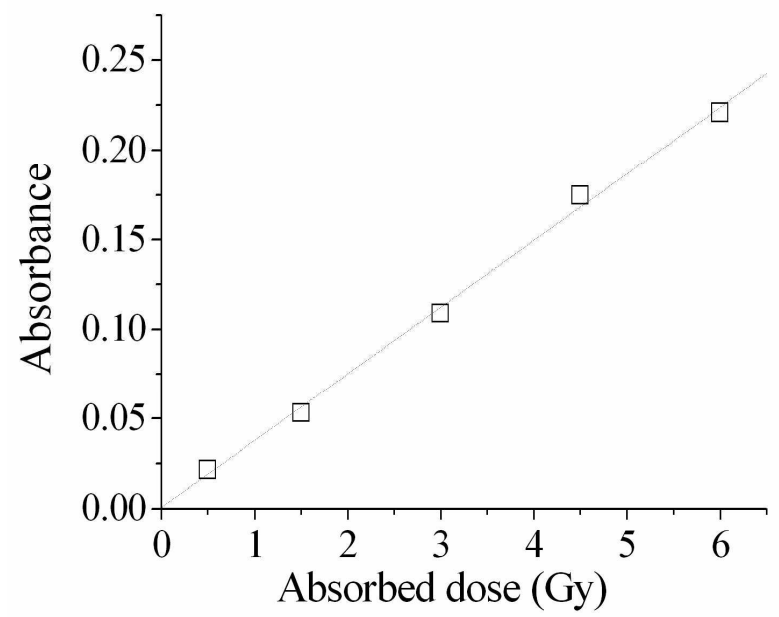

FIG. 2: FXG calibration curve (absorbance versus absorbed dose) for ${ }^{60} \mathrm{Co}$ energy photons, $10 \times 10 \mathrm{~cm}^{2}, 80 \mathrm{~cm} \mathrm{SSD}$ at the build-up depth $\left(\mathrm{R}^{2}=0.996\right.$ and first standard deviation $\left.=0.2 \%\right)$

\section{Output Factor}

The output factors measured with our FXG dosimeter appear in Fig.3 for $6 \mathrm{MV}$ and $10 \mathrm{MV}$ photons compared with ionization chamber measurements and with a Monte Carlo simulation.

The FXG dosimeter gives readings for small field sizes that are consistently higher than our $0.125 \mathrm{~cm}^{3}$ IC readings. The discrepancies between the FXG output factors and those from the ionization chamber for field sizes lower that $5 \times 5 \mathrm{~cm}^{2}$, correlate with the superior lateral spatial resolution of the FXG dosimeter.

Fig. 3a and $3 \mathrm{~b}$ show that the Monte Carlo simulation and the measurements made with the small ionization chambers do not significantly disagree with our FXG measurements. According to IAEA protocol [7], the dimensions of the detector should be less than $1 / 3$ of the field size dimension. Large ionization chamber shows larger discrepancies, when measuring small fields.

\section{Percentage Depth Dose}

The PDDs for square field sizes are presented in Fig. 4 (6 $\mathrm{MV}$ and $10 \mathrm{MV}$ ), results for FXG, $0.125 \mathrm{~cm}^{3} \mathrm{IC}, 0.1 \mathrm{~cm}^{3} \mathrm{IC}$ [10] and $0.1 \mathrm{~cm}^{3}$ IC [11]. From this figure, the PDD measurements in the $10 \mathrm{MV}$ set were multiplied by factor 2 (from the 

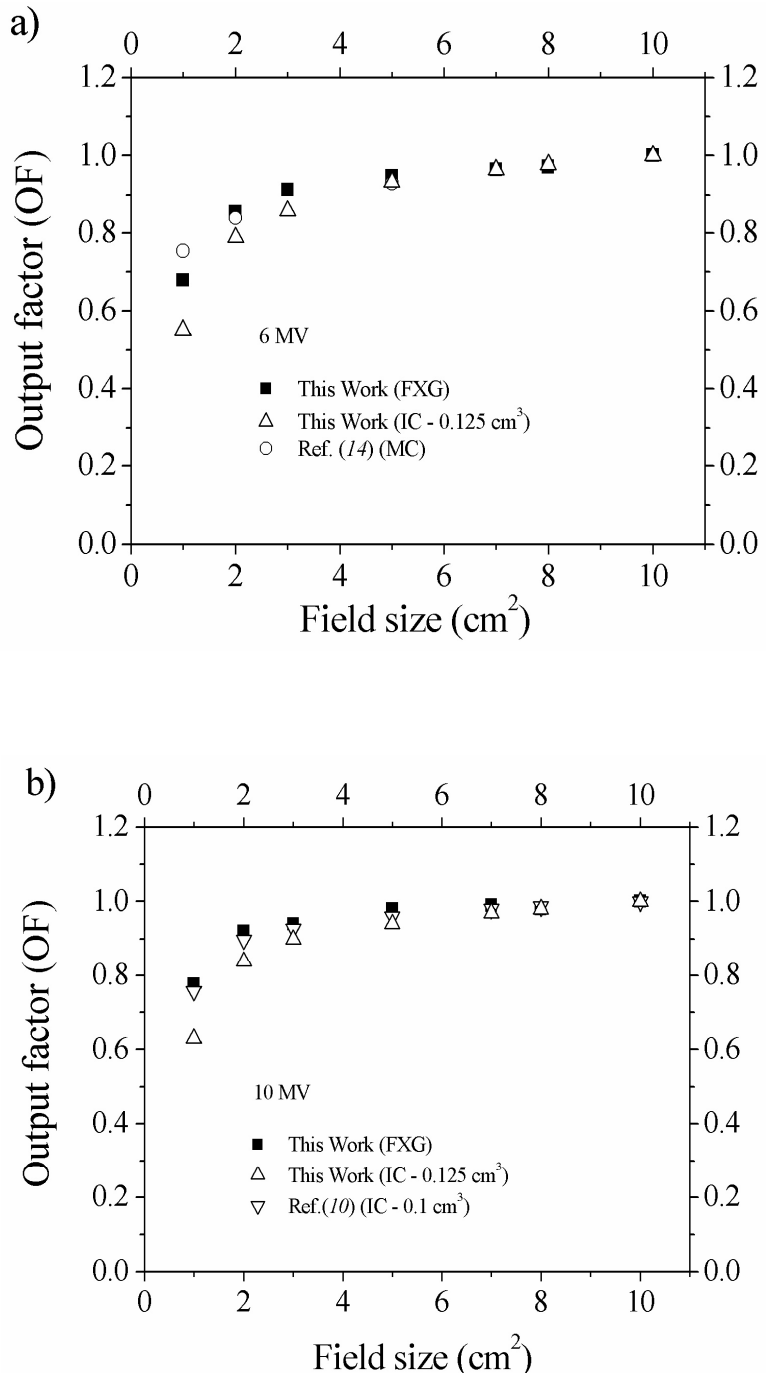

FIG. 3: Output factor versus square field size for 6 and $10 \mathrm{MV}$ photons at $100 \mathrm{~cm} \mathrm{SSD}$ and build-up depth. a) for $6 \mathrm{MV}(\square),(\triangle)$ and (O) are respectively for FXG, $0.125 \mathrm{~cm}^{3}$ IC and MC (14) (first standard deviation for FXG and $0.125 \mathrm{~cm}^{3} \mathrm{IC}=0.7 \%$ ) and $\mathrm{b}$ ) for 10 MV $(\square),(\triangle)$ and $(\nabla)$ are respectively for FXG, 0.125 and $0.1 \mathrm{~cm}^{3}$ IC (10) (first standard deviation for FXG $=2 \%$ and $0.125 \mathrm{~cm}^{3} \mathrm{IC}=$ $0.5 \%)$.

Eq. 3), in order that the values could be better visualized. All results (6 MV and $10 \mathrm{MV}$ sets) are displaced in accordance with the appropriate output factors, shown in Fig. 3, for the same reason.

In the two set of plots shown, one can see that the FXG dosimeter has enough resolution to show where the build-up depth occurs. Also, that all PDD values, obtained with the FXG, are more near to the values belonging to the smallest $\mathrm{IC}$, than for $0.125 \mathrm{~cm}^{3}$. One can also see that the difference between the dosimeters results increases when the field size diminishes. The PDD maximum difference between the FXG and the $0.1 \mathrm{~cm}^{3}$ IC [10] are $4.2 \%, 3.8 \%$ and $2.9 \%$ for $1 \mathrm{x}$ $1 \mathrm{~cm}^{2}, 3 \times 3 \mathrm{~cm}^{2}$ and $5 \times 5 \mathrm{~cm}^{2}$, respectively. These results can be explained through the followings facts: a) for smaller field sizes the lateral electronic equilibrium does not exist [1419], if the detector dimensions are less than $1 / 3$ of the field size dimension; b) the dosimeter can lose resolution due to the dose gradient $[17,20,21]$ and c) the photons energy spectra can be different of those used in standard laboratory for the IC calibration $[15,17,22]$.

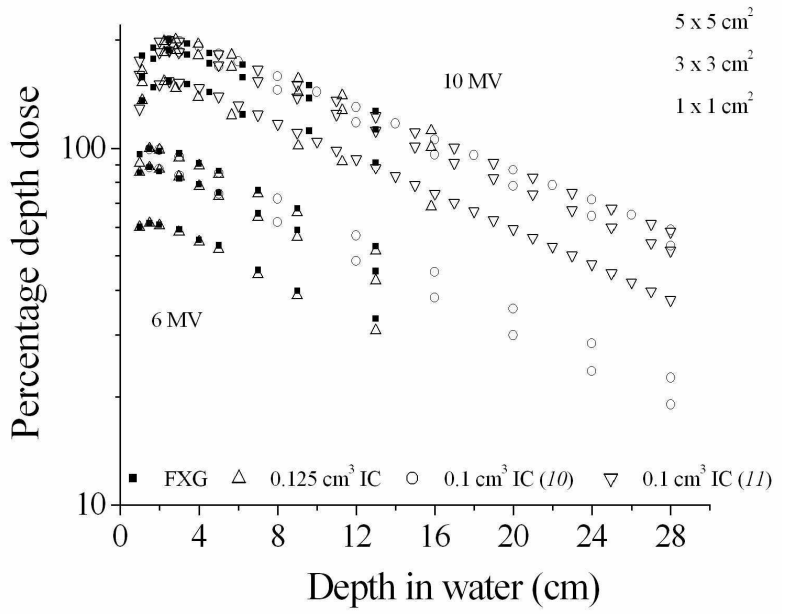

FIG. 4: PDD versus depth in water $(\mathrm{cm})$ for several square fields sizes $\left(1 \times 1,3 \times 3\right.$ and $\left.5 \times 5 \mathrm{~cm}^{2}\right)$ at 6 and $10 \mathrm{MV}$ photons, 100 $\mathrm{cm} \mathrm{SSD} \mathrm{(average} \mathrm{first} \mathrm{standard} \mathrm{deviation} \mathrm{for} \mathrm{FXG}=2.98 \%$ and for $\left.0.125 \mathrm{~cm}^{3} \mathrm{IC}=0.65 \%\right)$

\section{Field Profile}

The FXG and film profile behaviors for $6 \mathrm{MV}$ and $10 \mathrm{MV}$ are presented in Fig. 5. In the high gradient regions, the FXG values are near to those of film, once it also has an adequate spatial resolution for $3 \times 3 \mathrm{~cm}^{2}$ and $5 \times 5 \mathrm{~cm}^{2}$ field sizes.

According to anterior protocols [23-25] the field profile evaluations can be done considering three parameters: homogeneity, symmetry and penumbra.

The accepted tolerance for homogeneity is $\leq 2 \%$ of the difference between the lowest and the highest values. In our measurements a difference of the $1.00 \%$ was inferred for the $3 \times 3 \mathrm{~cm}^{2}$ and $5 \times 5 \mathrm{~cm}^{2}$, respectively for $6 \mathrm{MV}$ and $10 \mathrm{MV}$.

The accepted tolerance for symmetry is $\leq 2 \%$ of the difference between the left and the right values in $80 \%$ of the size, related to value at the central axis. In our measurements a difference of $1.90 \%$ was inferred for the $3 \times 3 \mathrm{~cm}^{2}$ and $5 \mathrm{x}$ $5 \mathrm{~cm}^{2}$, respectively for $6 \mathrm{MV}$ and $10 \mathrm{MV}$.

The accepted tolerance for penumbra is $\leq 2 \mathrm{~mm}$, distance differences of the points at $80 \%$ and $20 \%$ in region where the dose gradient is evaluated. In this study was observed a maximum deviation of the $0.92 \mathrm{~mm}$ between the two spatial 


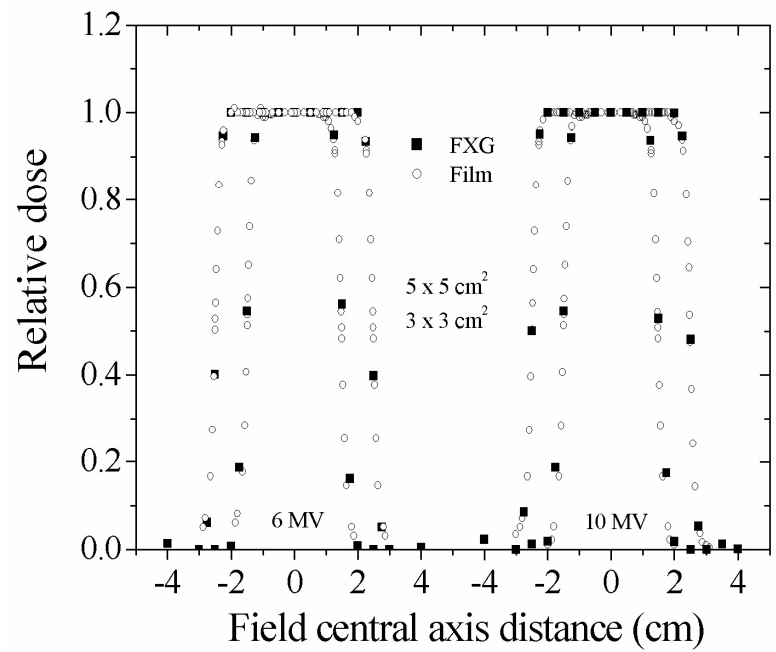

FIG. 5: Field size profiles from FXG ( $\square$ ) and X-OMAT film (O) dosimeters with $100 \mathrm{~cm} \mathrm{SSD,} 6$ and $10 \mathrm{MV}$ photons at their build-up depth. a) for $3 \times 3 \mathrm{~cm}^{2}$ and b) for $5 \times 5 \mathrm{~cm}^{2}$ (average first standard deviation for $\mathrm{FXG}=2 \%$ for both fields sizes).

positions of the FXG and film obtained for $3 \times 3 \mathrm{~cm}^{2}$ and $5 \mathrm{x}$ $5 \mathrm{~cm}^{2}$ field sizes and for 6 and $10 \mathrm{MV}$ photons.

The $\mathrm{RW}_{50}$ is the radiological width, expressed the difference between the calculated and measured dose distribution at $50 \%$ of the field gradient profile. In this study was observed a maximum deviation of $0.32 \mathrm{~mm}$ between the two $\mathrm{RW}_{50}$ (for FXG and film). This discrepancy obtained for $3 \times 3 \mathrm{~cm}^{2}$ and $5 \times 5 \mathrm{~cm}^{2}$ field sizes and $6 \mathrm{MV}$ and $10 \mathrm{MV}$, is within of the established tolerance $(<2 \mathrm{~mm})$.

The beam fringe, $\delta_{50-90 \%}$, expresses the distance between points at the $50 \%$ and the $90 \%$ of the field gradient profile. In this study was observed a maximum deviation of the 2.00 $\mathrm{mm}$ between the two spatial positions of the FXG and film dosimeters. This discrepancy obtained for $3 \times 3 \mathrm{~cm}^{2}$ and $5 \times 5$ $\mathrm{cm}^{2}$ field sizes and $6 \mathrm{MV}$ and $10 \mathrm{MV}$, is near to the established tolerance $(<2 \mathrm{~mm})$

\section{CONCLUSION}

The FXG, due to its atomic effective number and density near to those of the soft tissue, is expected to be an adequate dosimeter for radiotherapy, what can be confirmed through the dosimetric parameters of percentage depth dose, output factor and field profile. In this work, these parameters were obtained with the FXG and compared to the ionization chamber, film and Monte Carlo results.

In the small field sizes measurements, the FXG percentage depth dose and output factor results besides showing not significant differences with the others dosimeters and calculation method, permit one to infer respectively where the bild-up depth occurs and results more comparable to those from the smallest IC.

The FXG field profile results obtained, related to its homogeneity, symmetry and penumbra shown differences of 1.00 $\%, 1.90 \%$ and $0.92 \mathrm{~mm}$, respectively lower than accepted protocol tolerances $(\leq 2 \%)$, for the two first ones and $\leq 2$ $\mathrm{mm}$ for the last one.

Considering the dosimetric parameters results obtained, one can consider the FXG as an adequate dosimeter for radiotherapy, not only for conventional field sizes measurements, but also for radiosurgerical ones. In this way, we can conclude that this dosimeter can be used for general radiotherapy and radiosurgery control, through the dosimetric parameters measurements.

\section{Acknowledgments}

The present work was supported by grants from IAEA and CAPES (Brazil). The authors thank the Centro de Combate ao Câncer (CUCC-UERJ), the medical physicist Afranio Ackerman and technician José Luiz Aziani (DFM-FFCLRP-USP) for support.
[1] G. Gambarini, C. Birattari, M. Mariani, R. Marchesini, L. Pirola, P. Prestini, M. Sella, and S. Tomatis, Nucl. Instr. and Meth. in Phys. Res. B. 213, 321 (2003).

[2] N. V. Klassen, K. R. Shortt, J. Seuntjens, and C. K. Ross, Phys. Med. Biol. 44, 1609 (1999).

[3] W. I. D. Rae, C. A. Willemse, M. G. Lotter, J. S. Engelbrecht, and J. C. Swarts, Med. Phys. 23, 15 (1996).

[4] R. G. Kelly, K. J. Jordan, and J. J. Battista, Med. Phys. 25, 1741 (1998).

[5] M. A. Bero, W. B. Gilboy, P. M. Glover, and J. L. Keddie, Nucl. Instr. and Meth. In Phys. Res. B. 166, 820 (1999).

[6] R. T. Costa, Avaliando a viabilidade do uso do dosímetro Fricke modificado na radioterapia. [Master Thesis - Universidade de São Paulo, FFCLRP/DFM]. (2001).

[7] IAEA (Internacional Atomic Energy Agency) Absorved Dose Determination in External Beam Radiotherapy, Technical Reports series No. 398, (2000).
[8] F. M. Khan, The Physics of Radiation Therapy, Williams \& Wilkins, Baltimore (1997).

[9] AAPM A protocol for the determination of absorbed dose from high-energy photon and electron beams, Task Group 21, Med. Phys. 10, 741 (1983).

[10] P. V. Houdek, J. M. VanBuren, J. V. Fayos, Med. Phys. 10, 333 (1983).

[11] D. O. Findley, B. W. Forell, and P. S. Wong, Med. Phys. 14, 270 (1987).

[12] D. S. Felipe, Desenvolvimento, teste e aplicação de protótipo para dosimetria da solução Fricke modificada. [Master Thesis Universidade de São Paulo, FFCLRP/DFM] (2003).

[13] A. M. Caldeira Filho, Análise da solução Fricke modificada e irradiada através da espectroscopia fotoacústica. [Master Thesis - Universidade de São Paulo, FFCLRP/DFM] (2004).

[14] K. De Vlamynck, H. Palmans, F. Verhaegen, C. De Wagter, W. De Neve, and H. Thierens, Med. Phys. 26, 1874 (1999). 
[15] P. S. Nizin, Med. Phys. 26, 1893 (1993).

[16] A. Wu, R. D. Zwicker, A. M. Kalend, and Z. Zheng, Med. Phys. 20, 777 (1993).

[17] M. Heydarian, P. W. Hoban, and A. H. Beddoe, Phys. Med. Biol. 41, 93 (1996).

[18] I. A. Brezovich, P. N. Pareek, W. E. Plott, and R. L. S. Jennelle, Int. J. Radiation Oncology Biol. Phys. 38, 883 (1997).

[19] D. M. Duggan, C. W. Coffey, Med. Dosim. 22, 127 (1997).

[20] Y. W. Vahc, W. K. Chung, K. R. Park, J. Y. Lee, Y. H. Lee, O. Kwon, and S. Kim, Med. Phys. 28, 303 (2001).

[21] A. Mack, S. G. Scheib, J. Major, S. Gianolini, G. Pazmandi, H. Feist, H. Czempiel, and H. J. Kreiner, Med. Phys. 29, 2080 (2002).

[22] L. Núñez, F. Sánches-Doblado, Small field dosimetry in radio- surgery. Dynard, E.U. (1999).

[23] IAEA (Internacional Atomic Energy Agency), Commissioning and Quality Assurance of Computerized Planning Systems for Radiation Treatment of Cancer, Technical Reports series No. 430 (2004)

[24] G. J. Kutcher, L. Coia, M. Gillin, W. F. Hanson, S. Leibel, R. J. Morton, J. R. Palta, J. A. Purdy, L. E. Reisntein, G. K. Svensson, M. Weller, and L. Wingfield, Med. Phys. 21, 581 (1994).

[25] International Commission on Radiation Units and Measurements. Determination of absorbed dose in a patient irradiated by beams of $\mathrm{X}$ or gamma rays in radiotherapy procedures. Washington, USA: ICRU 24, (1976). 